Corugedo, Indalecio. Algunas reflexiones metodológicas sobre la evaluación costebeneficio de la sanidad. http://dx.doi.org/10.5209/rev_PADE.2012.n24.39544

\title{
ALGUNAS REFLEXIONES METODOLÓGICAS SOBRE LA EVALUACIÓN COSTE-BENEFICIO DE LA SANIDAD
}

\section{ON SOME METHODOLOGICAL ASPECTS OF THE COST-BENEFIT EVALUATION IN HEALTH ECONOMICS}

\author{
INDALECIO CORUGEDO DE LAS CUEVAS \\ Catedrático de Universidad \\ Departamento de Fundamentos del Análisis Económico I \\ Universidad Complutense de Madrid \\ icoruged@ccee.ucm.es
}

Fecha recepción: 19 de enero de 2012

Fecha aceptación: 9 de abril de 2012

\begin{abstract}
RESUMEN
La literatura reciente en la evaluación coste-beneficio de los proyectos sanitarios prefiere seguir la línea de la economía medioambiental y acercarse a una posición welfarista en la determinación de los cambios en el bienestar que continuar la hipótesis del capital humano, que había proporcionado en las últimas décadas unos notables resultados en los modelos de evaluación costebeneficio en la Economía de la Educación.
\end{abstract}

\section{Palabras Clave:}

Análisis coste-beneficio, inversiones sanitarias, capital humano, variaciones en el bienestar.

JEL: D61, I1, I2, I3.

\section{ABSTRACT:}

The recent literature on cost-benefit analysis in Health Economics normally follows the methodology accepted by the Environmental Economics rather than the classical Human Capital hypothesis adopted successfully by the Economics of Education. In this paper we try to argue what could be the main reasons for such an investigation line.

\section{KEYWORDS:}

Cost-Benefit Analysis, Investment in Health, Human Capital, Welfare Changes. 
Corugedo, Indalecio. Algunas reflexiones metodológicas sobre la evaluación costebeneficio de la sanidad.

\section{INTRODUCCIÓN}

La consideración de los estudios económicos sobre el sector sanitario como un área específica en la literatura económica es bastante reciente. El economista ha tardado en entrar a estudiar los hechos clínicos desde su perspectiva particular, es decir, bajo la consideración de un problema de asignación óptima de recursos escasos. Existen ciertas áreas en donde existen numerosos y, en ocasiones, insalvables problemas de definición y valoración de los factores productivos y de los bienes y servicios producidos a partir de aquellos, lo que confiere una mayor dificultad a la hora del estudio económico basado en términos de oferta y demanda de los agentes económicos implicados. Esto ha ocurrido, por ejemplo, en las áreas que estudian la economía aplicada al medio ambiente, a la educación y a la sanidad. Las dificultades dan pie a la aparición de corrientes alternativas a la hora de resolver los problemas. Por ello vamos a comenzar distinguiendo aquí dos corrientes metodológicas claramente definidas, la welfarista, desarrollada por las economías del medioambiente y de la salud, y la que utiliza la hipótesis del capital humano, aplicada tradicionalmente por el área de la economía de la educación. Si bien ambas corrientes metodológicas no son incompatibles, los modelos que proporcionan ofrecen un desarrollo y resultados claramente diferenciados. En los párrafos siguientes vamos a tratar de hacer primero una breve explicación de cada una de estas dos tendencias y luego una serie de reflexiones de las razones de una adopción mayoritaria de la hipótesis welfarista por parte de los economistas del área sanitaria.

\section{LAS VARIACIONES EN EL BIENESTAR COMO OBJETIVO}

Si atendemos al diseño de un modelo teórico para la optimización de los recursos su aplicación empírica se ajusta perfectamente a la conocida metodología del análisis coste-beneficio. Si existen una serie de recursos disponibles destinados a la realización de una serie de necesidades lo inmediato es establecer un proceso de priorización entre las alternativas disponibles para la satisfacción de aquellas necesidades. Una regla económica básica de comportamiento sería establecer una ordenación basada en los beneficios netos derivados de cada una de las alternativas. Sería así prioritaria la solución con unas ventajas netas más elevadas. Pero hablar de ventajas o de beneficios implica hablar de unos ingresos, derivados de las acciones de demanda, y de unos costes, derivados de las acciones de oferta de los agentes implicados en los procesos productivos. En este argumento identificamos beneficio con variaciones en el bienestar, con lo que analizaremos las diferentes alternativas en términos del bienestar proporcionado.

La consideración del bienestar económico ha dado lugar a toda una literatura dentro del análisis microeconómico. Hablar de mejoras individuales ha supuesto la introducción en el análisis de un conjunto interminable de juicios de valor que ha conseguido engrosar una de las áreas más sospechosas sobre su carácter científico dentro del análisis microeconómico, la llamada Economía del Bienestar. Lejos de entrar en las polémicas sobre las diferentes alternativas que se ofrecen para la medición del bienestar individual y/o social, nos limitaremos aquí a un sencillo concepto, el de excedente del consumidor. 
Corugedo, Indalecio. Algunas reflexiones metodológicas sobre la evaluación costebeneficio de la sanidad.

La lógica económica elemental para la evaluación de un determinado proyecto o alternativa establecería una primera consideración de los beneficios brutos que el proyecto proporcione a sus consumidores potenciales. El término económico más adecuado aquí sería el de disponibilidad a pagar por el proyecto por parte de sus usuarios futuros. Se trata aquí del análisis de una demanda potencial (Stated preferences) generada por los supuestos consumidores futuros. Pero esta demanda genera igualmente unos costes, sobre todo representados por el coste de oportunidad de la elección. El llamado excedente del consumidor representaría entonces el beneficio neto de adoptar una alternativa, en términos de la diferencia entre los beneficios brutos y costes señalados anteriormente.

Los recientes análisis de evaluación económica de la sanidad adoptan esta metodología cuando los beneficios netos se expresan en términos monetarios (análisis coste-beneficio), si bien desde los orígenes de la evaluación económica en el área de la salud se han empleado técnicas menos exigentes, representadas fundamentalmente por el análisis coste-efectividad/coste utilidad que, aunque es verdad que ha ofrecido modelos para la evaluación muy meritorios, se aparta sustancialmente de lo que es una auténtica evaluación económica.

Un ejemplo típico que se plantea en una evaluación coste-beneficio en sanidad es cuando se trata de valorar la entrada de un fármaco nuevo en el mercado. Se trata en primer lugar de determinar los beneficios privados y sociales brutos derivados de la introducción del fármaco, es decir, la disposición a pagar por el fármaco. Es claro que la dispersión en las preferencias de los diferentes individuos por el fármaco será muy significativa. No solo podemos hablar de una notable dispersión de facto entre los diferentes individuos sino una segunda dispersión a la hora de la revelación de las preferencias por parte de aquellos. Todo ello supone que la disponibilidad a pagar como medida de la demanda o de los beneficios brutos está sometida a unos notables sesgos por parte de los consumidores.

Por ello muchos de los evaluadores en el área de la Economía de la Salud se han conformado con valorar las ventajas derivadas de un nuevo fármaco o de una nueva tecnología sanitaria calculando su efectividad en términos de los años de vida ganados por la nueva inversión (análisis coste-efectividad) o de tales años ganados ponderados por un cierto índice de la calidad de vida del paciente (análisis coste-utilidad). En este último caso se define una nueva unidad de medida de la utilidad de los individuos, el AVAC (años de vida ganados ponderados por la calidad de vida). De esta forma es cierto que se evitan las consideraciones sobre unas adecuadas medidas del bienestar en términos monetarios. Se podría entonces sintetizar el estado de la literatura sobre evaluación sanitaria en una posición esencialmente welfarista, basada en medidas de bienestar dadas por la ganancia neta de años de vida, la utilidad generada por esos años de vida adicionales o, en términos de una mayor rigurosidad económica, por un incremento del bienestar en términos monetarios dado por los correspondientes excedentes del consumidor, variaciones equivalentes y variaciones compensatorias de renta. 
Corugedo, Indalecio. Algunas reflexiones metodológicas sobre la evaluación costebeneficio de la sanidad.

Un nuevo tipo de modelos que deberían ser agregados al desarrollo de esta vía de evaluación en términos de bienestar sería el llamado salarios ajustados por la calidad de vida (SAVAC) (Corugedo et al. 2007). En estos modelos no se utiliza la demanda compensada de un bien para valorar las variaciones de bienestar derivadas de las inversiones sanitarias sino la oferta compensada de trabajo. Estos modelos están basados en aquellos modelos de oferta de trabajo con impuestos, en donde era posible la determinación de las variaciones en el bienestar derivadas de modificaciones en los tipos impositivos del Impuesto Personal sobre la Renta de las personas físicas.

El supuesto principal en el que se sustenta este tipo de modelos es la consideración de una enfermedad como un impuesto (health tax). Así de la misma manera que un impuesto directo de tipo proporcional rebaja las rentas salariales disponibles de los individuos, disminuyéndose la capacidad de consumo y el bienestar, una enfermedad (migraña) puede determinar unos resultados análogos en las rentas salariales de los individuos que la padecen. En el caso de la imposición sobre la renta, el individuo pierde efectivamente parte de su renta disponible, cosa que no ocurre para el caso de una enfermedad. Sin embargo la renta potencial o renta disponible para el consumo por el individuo enfermo puede ser equivalente a la renta disponible fiscal anterior.

Lo importante aquí es fijar el índice que ha de representar a un cierto tipo impositivo $t$ en los modelos SAVAC. Tras una serie de estudios piloto realizados para el caso de pacientes con migraña se concluyó que un índice de calidad de vida $\boldsymbol{i}$ tipo EuroQol $5 D$ podía considerarse equivalente al tipo impositivo buscado. De esta forma la operatividad sería la siguiente. Si un individuo sano recibe un salario $W$ una determinada enfermedad puede rebajar su salario potencial o salario ajustado por una cierta calidad de vida a un nivel $W^{\prime}=W i$ tomando el índice elegido los valores $0 \leq i \leq 1$. De esta forma un individuo totalmente sano $(i=1)$ permanecería con un salario potencial o un salario ajustado por la calidad de vida equivalente a su salario actual.

El modelo SAVAC ha sido utilizado en estos últimos años para la evaluación de las variaciones en el bienestar derivadas de diferentes patologías, tales como la migraña, la esquizofrenia, y sus tratamientos e igualmente para medir las ganancias de bienestar derivadas de diferentes intervenciones quirúrgicas, tales como la vertebroplastia y la cifoplastia, que se aplican a pacientes que han sufrido procesos de fracturas vertebrales de diferentes tipos y orígenes. Ambas intervenciones restablecen inmediatamente la altura vertebral de los pacientes y existe una rápida y notoria mejora de la calidad de vida.

El modelo precisa de una primera etapa en la que se trata de determinar la calidad de vida del paciente antes de la intervención, reflejada por el correspondiente valor $i_{0}$ del índice EuroQol. En una segunda etapa se calcula el valor de este mismo índice tras la intervención quirúrgica $i_{1}$ y se aplican ambos valores a un modelo fiscal de determinación de variaciones del bienestar cuando el tipo impositivo proporcional sobre la renta cobra los valores anteriores. Se trata, en definitiva, de determinar el área de la Figura 1. 
Corugedo, Indalecio. Algunas reflexiones metodológicas sobre la evaluación costebeneficio de la sanidad.

Antes de la intervención el paciente se encuentra en la situación $A^{\prime}$, en donde la enfermedad le determina poder trabajar únicamente un número de horas $L^{\prime}$ a un salario potencial reducido en Wi unidades monetarias. La intervención le restablece en una posición $A$, con una posibilidad de aumento de aumento del número de horas trabajadas en $\left(L-L^{\prime}\right)$ y un aumento del bienestar igual al área sombreada.

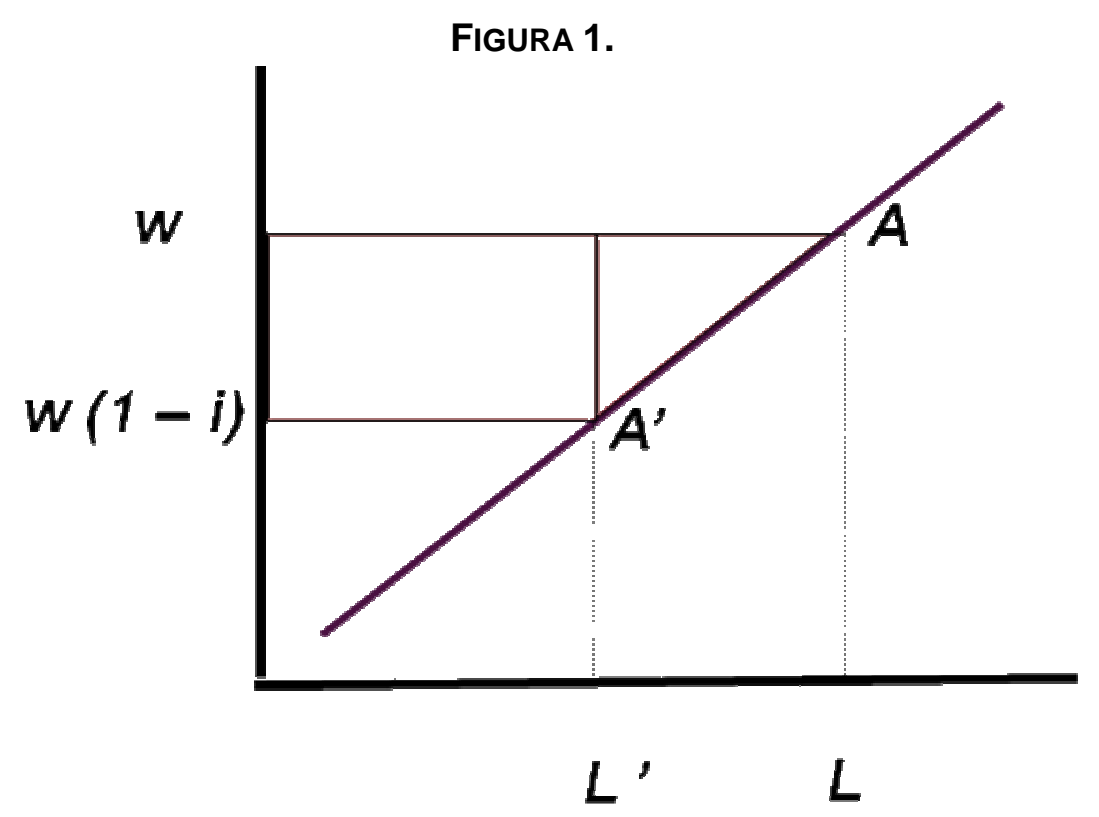

El salario ajustado por la calidad de vida supone un avance respecto al $A V A C$, años de vida ganados ajustados por la calidad de vida, medida utilizada normalmente en los análisis de evaluación tipo coste-efectividad. Se podría decir que la medida que aquí se introduce, el SAVAC supone, en cierto modo, una monetarización de los $A V A C$ a través de los salarios.

El valor añadido, entonces, del modelo que aquí se presenta, en términos de la literatura sobre la evaluación de las inversiones sanitarias se podría concretar en las tres observaciones siguientes:

- Un modelo basado en los SAVAC como medida de evaluación de resultados supone un avance en el análisis coste-efectividad/coste-utilidad, ya que ofrece un resultado en unidades monetarias, característico de un análisis coste-beneficio

- Este modelo ofrece una perspectiva a corto plazo y trata de evaluar el presenteismo o pérdida de productividad asociada a una enfermedad sin que existan bajas laborales. Se aparta así de aquellos modelos que miden las pérdidas de productividad como pérdidas de capital humano (absentismo) reflejadas en las bajas laborales debidas a la enfermedad, lo que supone ya un tratamiento económico en términos del largo plazo.

- Por último, aún teniendo en común la expresión de un resultado en términos de una valoración monetaria de las variaciones en el bienestar, reflejada por el excedente del consumidor o por la correspondiente variación equivalente de la renta, igual que lo hace el método de la variación contingente como medida de una cierta disponibilidad a pagar por parte del público, el modelo basado en los SAVAC utiliza el salario de mercado como unidad básica de 
Corugedo, Indalecio. Algunas reflexiones metodológicas sobre la evaluación costebeneficio de la sanidad.

medida, más robusta generalmente que lo que unos individuos puedan revelar como su disponibilidad a pagar por las alternativas que se les ofrecen.

Para el desarrollo del modelo y la obtención de resultados se distinguirán dos etapas:

1) Cálculo del índice de calidad de vida (EuroQol 5-D) correspondiente al estado de salud que se pretende evaluar. Esto se puede hacer a dos niveles, bien a un nivel micro totalmente desagregado por individuos o bien a un nivel agregado representado por un índice de calidad medio de la muestra de individuos. En ambos casos el índice se puede deducir por la tabulación de las respuestas al cuestionario correspondiente y/o por la llamada escala visual analógica (VAS). Con la determinación de estos índices de calidad de vida se puede establecer ya una distribución de los índices según diferentes enfermedades y diferentes tratamientos. Es muy probable que, aún tratándose de patologías y tratamientos diferentes, el estado general de salud del individuo sea equivalente.

2) Estimación de una función de oferta de trabajo que explique el comportamiento laboral de una economía ante cambios en los parámetros de la función. Las situaciones en salud de los individuos a valorar han de pertenecer a esta función, es decir, los puntos que indiquen estados de salud diferentes han de estar sobre la curva de oferta de trabajo. Tal curva ha de ser una curva de oferta compensada, pues es la expresión requerida para la curva de oferta de trabajo para poder medir sobre ella variaciones en el bienestar. (Ver figura). Para una obtención directa de las variaciones en el bienestar debidas a modificaciones en las políticas fiscales se utiliza el modelo SIRPIEF (Sanz, J.F. 2005). Se trata de un modelo de simulación para la estimación de las variaciones en el bienestar y alteraciones en decisiones laborales, tales como la participación y/o el número de horas de trabajo ofrecidas. Las variaciones en el bienestar están expresadas en el modelo SIRPIEF por las correspondientes variaciones equivalentes de rentas que expresan, en términos ahora de modificaciones laborales, lo que los individuos estarían dispuestos a pagar para alcanzar las mejoras en el bienestar con el tratamiento médico. Todo ello supone, en definitiva, una forma más robusta de calcular la disponibilidad a pagar por parte del público.

Los resultados de este modelo aplicado al estudio de la olanzapina, que se trata de un principio activo muy efectivo para el tratamiento de la esquizofrenia son los siguientes:

La aplicación que se hace aquí del modelo se basa en el Schizophrenia Outpatient Health Outcomes (SOHO) study. Se trata de un estudio observacional prospectivo a largo plazo (3 años) sobre tratamientos de la esquizofrenia en 10 países europeos: Dinamarca, Francia, Alemania, Grecia, Irlanda, Italia, Holanda, Portugal, España y el Reino Unido). La población del estudio está formada por pacientes externos con esquizofrenia, bien que inician la terapia por primera vez o bien que cambian de terapia. Se enrolaron inicialmente para el estudio 10.972 pacientes, de los que tras una selección, se trabajó con una muestra final de 10.205 pacientes. 
Corugedo, Indalecio. Algunas reflexiones metodológicas sobre la evaluación costebeneficio de la sanidad.

Los pacientes padecen la enfermedad con una media de duración de 11.3 años y la mayoría son hombres (57.8\%). A partir de esta muestra general se obtiene, para la aplicación del modelo, una nueva selección de pacientes, correspondientes a los países que ofrecen un mayor número de individuos que están empleados en el momento del estudio. Esto supone la consideración de únicamente cuatro países, España, Francia, Alemania e Italia que, además presentan una elasticidad de sustitución semejante en la oferta de trabajo. Todo ello reduce a 1060 el número de individuos que entran en consideración para el modelo.

Si se calculan los valores medios del índice de calidad de vida antes y después del tratamiento controlado y a continuación se calculan las variaciones de este índice de calidad de vida, los resultados son los siguientes (Prieto, L. et al, 2003):

\section{$\Delta \mathrm{i}=0.18$}

Para la estimación directa de las variaciones equivalentes de renta a través de la oferta de trabajo según el modelo SIRPIEF se utiliza una muestra de empleados de la UE-15 de 46.046 individuos según los datos PHOGUE correspondientes al comportamiento de las economías domésticas con referencia al año 2000. Se consideran únicamente las personas empleadas, por lo que quedan omitidos de la muestra los grupos de estudiantes, empleados por cuenta propia y jubilados.

Si los resultados sobre variaciones en los índices de calidad de vida anteriores se aplican al modelo de oferta de trabajo, tenemos los resultados agregados siguientes:

\section{CuAdRO 1. VARIACIONES EQUIVALENTES PARA LOS TRATAMIENTOS DE ESQUIZOFRENIA} POR PAÍSES. EUROS POR AÑO.

\begin{tabular}{|l|l|}
\hline España & $1.521,81$ \\
\hline Francia & $1.171,21$ \\
\hline Alemania & $1.407,52$ \\
\hline Italia & $1.268,14$ \\
\hline Valor medio & $\mathbf{1 . 3 4 2 , 1 7}$ \\
\hline Fuente: elaboración propia.
\end{tabular}

La interpretación económica de los resultados anteriores es que el tratamiento evaluado significa para el paciente una mejora anual media de su bienestar de $1.342,17 €$, lo que puede interpretarse como un cálculo de los beneficios netos (excedente del consumidor) del tratamiento. El cálculo incluye ya los costes indirectos del tratamiento.

\section{LAS VARIACIONES EN EL CAPITAL HUMANO COMO OBJETIVO}

Una forma alternativa de valorar los resultados de la aplicación de tecnologías sanitarias alternativas y de la administración de medicamentos contempla la atención sanitaria explícitamente como una inversión en salud. La 
Corugedo, Indalecio. Algunas reflexiones metodológicas sobre la evaluación costebeneficio de la sanidad.

consideración económica de la inversión en asistencia sanitaria equipara esta vía metodológica a la inversión en educación y sigue la trayectoria tradicional de la hipótesis del capital humano. La educación es un flujo que aumenta el stock de capital humano en los individuos, tal que aumenta la productividad en aquellos. El arranque de esta hipótesis está en Schultz (1960) y se desarrolla plenamente con Mincer (1974). Análogamente en nuestro caso la atención sanitaria supone un flujo que incrementa un stock de capital humano en forma de salud. Esta hipótesis alternativa permite distinguir los dos bienes claramente diferenciados utilizados por la literatura de la Economía de la Salud, la salud y la atención sanitaria.

Bajo el desarrollo de esta hipótesis el análisis coste-beneficio no se centraría, como en el caso visto en el epígrafe anterior, en una evaluación de unas variaciones en el bienestar representadas por el excedente del consumidor, sino en una medida del incremento del capital humano determinado por la aplicación a los pacientes de atenciones sanitarias alternativas 0 de medicamentos de nueva presencia en el mercado. Un incremento de la salud viene reflejado luego por un incremento de los salarios.

Supongamos que una Comunidad Autónoma invierte en una campaña de vacunación antigripal. Existirán unos costes y unos beneficios derivados de la campaña. Aquí se hace evidente el carácter de bienes semi-públicos de la salud y la atención sanitaria. En tal inversión se distinguirán unos costes privados, que en este caso serían nulos si la vacunación corre a cargo del Sistema Nacional de Salud, y unos costes sociales, reflejados por los costes directos derivados de la vacunación y el coste de oportunidad o coste derivado del tiempo empleado en llevarla a cabo. Igualmente ocurrirá con los beneficios. Los beneficios privados los recogerán los individuos, en términos de una disminución de la probabilidad de contraer la enfermedad y por lo tanto de incurrir en bajas laborales. Los beneficios sociales están igualmente recogidos por el aumento de la productividad en la Comunidad al disminuir la probabilidad de existencia de procesos gripales entre sus ciudadanos.

Existen habitualmente dos formas alternativas de registrar los beneficios netos derivados de inversiones en capital humano. Supongamos que un paciente de trastornos renales crónicos es sometido a un trasplante de riñón. Podemos considerar que se trata de una inversión sanitaria tal que el paciente espera una mejora en su estado de salud y en su productividad a lo largo de una serie de años. Se podría aquí definir un cierto Valor Actual Neto (VAN) de los beneficios netos esperados futuros debidos al trasplante, definido por:

$$
V A N=\Sigma B_{i} /(1+r)^{i}
$$

siendo $B_{i}$ los beneficios netos esperados por el paciente a lo largo de los $i$ periodos de esperanza de vida ( representados por las rentas netas esperadas durante el ciclo vital) y $r$ una tasa de descuento que represente una aproximación al coste de oportunidad del trasplante.

La valoración de los costes y los beneficios por este procedimiento es cierto que podría determinar decisiones poco justas, pues ante la igualdad de otros 
Corugedo, Indalecio. Algunas reflexiones metodológicas sobre la evaluación costebeneficio de la sanidad.

factores, las decisiones de inversión se inclinarían hacia los pacientes más jóvenes, que presentan un horizonte temporal de vida más amplio. Esto puede suponer una de las razones por las que la hipótesis del capital humano no ha terminado de ser aceptada en el mundo sanitario de una manera tan amplia como lo fue en la economía de la educación.

Una valoración de la aportación de las inversiones sanitarias al capital humano de los individuos que las realizan podría venir dada por un modelo dado por la ecuación semilogarítmica siguiente (Mincer, 1974)

$$
\log W=a+b A S+c S+d A+e Z+u
$$

siendo $W$ las rentas salariales, $A S$ la inversión en atención sanitaria, $S$ una variable que represente el nivel educativo del individuo, $A$ representa la habilidad, inteligencia o capacidad innata para producir del individuo y $Z$ supone un conjunto de variables exógenas que recogen el entorno familiar y social del individuo. La ecuación anterior recoge así la productividad o capital humano agregado de un individuo (explicado por sus rentas laborales) en términos de todos los componentes educativos y sanitarios que lo generan. En particular los estimadores mínimo-cuadráticos de los parámetros $b$ y $c$ indicarían las aportaciones marginales respectivas de la sanidad y la educación a las rentas laborales de un individuo.

Por último, dediquemos el resto de este artículo a la introducción de un papel cada vez más relevante de la sanidad en la economía de un país. Nos vamos a referir al significado de la sanidad como señal o la sanidad como filtro.

En 1974 Michael Spence introduce los mecanismos de signalling y screening en aquellas economías sujetas a situaciones de información asimétrica. En un mercado es muy corriente que los oferentes tengan una información más completa que los demandantes sobre las características de los bienes que pretenden intercambiar. Unos años antes, Akerlof (1970) ya hablaba de los problemas de la falta de información de los mercados en su mercado de coches de segunda mano. Los vendedores de coches conocen la calidad de los vehículos, cosa que no ocurre con los compradores. Si no se recurre a mecanismos que revelen la calidad de los objetos que se pretenden intercambiar se producirán nuevas ineficiencias en los mercados debidas a esta escasez de información. En el mercado de coches usados de Akerlof los propios precios pueden actuar como señales sobre las calidades de los coches. Un precio excesivamente bajo (una ganga) puede ser una señal de mala calidad, la demanda se retrae y puede llegar a desaparecer el mercado. Para Spence la señalización se realiza a través de la educación y existen dos tipos de señalización llamados signalling o screening según se produzca por el lado de la oferta o de la demanda. Hablar de señalización es hablar del establecimiento de un mecanismo de separación, filtro o discriminación entre individuos con diferente capacidad productiva (calidad).

La señalización se produce de la siguiente forma. Supongamos que un seleccionador de recursos humanos de una empresa se enfrenta a un grupo de individuos (trabajadores potenciales) con dos niveles diferentes de 
Corugedo, Indalecio. Algunas reflexiones metodológicas sobre la evaluación costebeneficio de la sanidad.

productividad. Los individuos conocen su propia productividad, pero no ocurre lo mismo con quien pretende contratarles. Si no existe un proceso de señalización previo al contrato y todos son tratados por igual con la asignación de un salario único, habrá unos incentivos a no entrar en el mercado por parte de los individuos pertenecientes al grupo de una alta productividad. El mercado se quedaría entonces con los individuos poco productivos, recibiendo además un salario más alto que el que correspondería a su nivel de productividad. Esta ineficiencia del mercado es conocida como un fenómeno de selección adversa. Pero para que exista el proceso de separación de individuos se requiere un supuesto básico de comportamiento. Los costes de selección han de ser mayores para el grupo de individuos de una baja productividad. El equilibrio separador que establece todo mecanismo de señalización procede de que son los propios individuos los que tienen incentivos o no para ser señalizados. Supongamos que la señalización la establece un título académico. Para los individuos de baja productividad los costes de conseguir el título serían superiores a los beneficios que suponen su propia señalización. De este modo el título (educación) ha actuado como filtro entre individuos de diferente productividad. El llamado screening es un mecanismo simétrico al anterior que se produce por el lado de la demanda. Un seleccionador de recursos humanos tiene la experiencia de que una determinada titulación es indicativa de un cierto nivel de productividad. De este modo, cada tipo de señal determinará una cierta probabilidad de empleo junto con un cierto salario (self confirming mechanism).

Pasemos ahora al mercado sanitario. Los ejemplos de información incompleta y asimétrica y los mecanismos de signalling y screening aparecen por todas partes. Empecemos por una compañía de seguros médicos que trata de determinar unas primas justas para sus asegurados. Si existe una prima única para todos los asegurados se generan incentivos para abandonar el mercado (no se aseguran) aquellos individuos con una probabilidad baja de contraer una enfermedad, como los jóvenes. Si no existiese una señalización previa, de forma que se pueda establecer una discriminación entre las primas, operaría un mecanismo de selección adversa, tal que la compañía de seguros se quedaría con aquellos individuos más proclives a estar enfermos. Por ello una compañía de seguros médicos establece primas más altas según la edad y la cronicidad de enfermedades y requiere al potencial asegurado una cierta información sobre su currículum sanitario.

En el mundo sanitario existe en un principio una asimetría en la información entre médicos y pacientes. Éstos tienen en un principio un conocimiento de su currículum sanitario que el médico no posee. Por ello una primera consulta supone el comienzo del funcionamiento de unos mecanismos de screening que tratan de revelar el estado de salud de un paciente. Este es el motivo por el que se pueda establecer el supuesto de que una Atención Primaria sea no solo un filtro (Ortún, 1998) o entrada de un paciente en el sistema sino el lugar en donde comienzan a desarrollarse unos mecanismos de oferta (signalling) y demanda (screening) de señales.

La eficiencia de un sistema de Atención Primaria se puede analizar entonces en términos del buen funcionamiento de los mecanismos de señalización anteriores. Podemos considerar que ambos mecanismos actúan 
Corugedo, Indalecio. Algunas reflexiones metodológicas sobre la evaluación costebeneficio de la sanidad.

simultáneamente, pero atendiendo al aspecto temporal los mecanismos de oferta serían previos a los mecanismos de demanda. Un individuo que sospecha tener una enfermedad acude a la Atención Primaria si evalúa unos costes de la asistencia (directos e indirectos) inferiores a los beneficios (la revelación de información sobre la posible enfermedad). Si la atención sanitaria es pública los costes directos serían nulos, reduciéndose entonces la partida a los costes del transporte y, sobre todo, al coste de oportunidad del tiempo que emplea en la asistencia. Para un buen funcionamiento de la Atención Primaria en términos de filtro del sistema es, por ello, esencial, establecer mecanismos de disuasión para evitar que acudan al sistema aquellos individuos que realmente no lo necesitan, bien porque no están enfermos o porque la levedad de su enfermedad no requiere asistencia sanitaria alguna. Dos mecanismos alternativos de disuasión podrían ser el copago o el pago por consulta con posterior reembolso (Francia). Existen puntos de vista muy diferentes sobre la eficiencia de estas dos medidas, pues introducen una complejidad adicional en la administración y son bastante discutibles en términos de equidad. En el caso español el mayor problema que existe para la eficiencia de la Atención Primaria es la dispersión del médico en tareas de tipo administrativo. Si el tiempo medio establecido por la Unión Europea para la dedicación clínica a cada paciente es de 15 minutos, en la mayoría de las Comunidades españolas este tiempo no excede de 3 minutos, con lo que desaparece entonces el papel esencial que tiene este tipo de atención.

Una vez que un individuo asiste a un centro médico se convierte en paciente del sistema y es ahora cuando funcionan los mecanismos de screening. En esta fase se pueden incluir análisis de todo tipo, radiologías y cualquier tipo de técnica que permita hacer un primer diagnóstico. La ya citada escasez de tiempo que tiene un médico de primaria a realizar este screening deriva esta actividad en muchos casos hacia la Atención Especializada colapsando aquella. En la Atención Primaria debería realizarse con toda seguridad un primer diagnóstico en los términos siguientes:

1) ¿Está el individuo realmente enfermo?

2) Si lo está, ¿puede ser tratado en el centro o es necesario su envío a un especialista?

Un buen diagnóstico inicial requiere que, además de la disposición por parte del médico del tiempo necesario para realizarlo, los centros dispongan de una tecnología adecuada, exista una adecuada organización del trabajo y el personal médico disponga de un adecuado y competente personal de enfermería, elementos de los que carece el actual Sistema Nacional de Salud español.

\section{CONCLUSIONES}

El trabajo del economista se hace cada vez más necesario en el mundo sanitario en una situación histórica en donde cada vez se hace más relevante una buena asignación y organización de los recursos escasos. Este trabajo requiere una fase previa de priorización de necesidades que conduce a la necesidad de hacer una buena evaluación económica de todas las alternativas disponibles para alcanzar el objetivo económico deseado. El análisis coste- 
Corugedo, Indalecio. Algunas reflexiones metodológicas sobre la evaluación costebeneficio de la sanidad.

beneficio nuevamente se plantea como aquella metodología que introduce fundamentalmente el sentido común en el mundo de la política económica sanitaria. Pero ya se ha señalado en este artículo que en otras áreas como la educación y el medioambiente la valoración de los resultados en términos de unidades monetarias, ya sean en forma de salarios (capital humano) o de excedentes del consumidor (welfarismo) se ha llevado a cabo de una manera mucho más rápida y efectiva. Muchos investigadores en el área sanitaria consideran casi una aberración que un estado de salud individual pueda ser medido en euros cuando, a efectos prácticos, los presupuestos de todo tipo son recogidos en términos monetarios y la unidad monetaria no tiene un sentido alternativo al de una simple consideración de unidad de cuenta.

Estas breves reflexiones sobre la metodología básica de esa nueva parcela de la microeconometría Ilamada Economía de la Salud pretenden sobre todo llamar la atención sobre la importancia creciente que tiene la economía en los procesos que pretenden realizar una óptima asignación de los recursos sanitarios. Médicos y economistas han de compartir en el futuro los mismos caminos. 
Corugedo, Indalecio. Algunas reflexiones metodológicas sobre la evaluación costebeneficio de la sanidad.

\section{REFERENCIAS BIBLIOGRÁFICAS}

Akerlof, G. (1970): "The market for 'lemons': Quality uncertainty and the market mechanism", Quarterly Journal of Economics, 84, 3, pp. 488-500.

Corugedo, I., Antón, E., Hidalgo, A. y Del Llano, J. (2007): "Towards a new model for the evaluation of welfare changes in Health Economics", European Journal of Health Economics, 7, 1.

Mincer, J. (1974): Schooling, Experience and Earnings. NBER.

Ortún, V. (2011): Refundación de la Atención Primaria. Springer Healthcare.

Prieto, L., Dilla, T., Ciudad, A. y Sacristan, J. A. (2003): "Olanzapina: Un tratamiento coste-efectivo para pacientes con esquizofrenia", Revista Española de Economía de la Salud.

Sanz, J. F. (2007): Manual del SIRPIEF. Instituto de Estudios Fiscales.

Schultz, T. (1961): "Investment in Human Capital", The American Economic Review, 51, 1, pp. 1-17.

Spence, M. (1974): “Job Market Signaling”, Quarterly Journal of Economics, 87, 3, pp. 355-374. 